\title{
Developing a social media strategy for R\&D in energy efficiency - a case study in progress
}

\author{
Elmar Bartlmae ${ }^{1, *}$, Luis Arboledas-Lérida ${ }^{1}$, and Natalie Höppner ${ }^{1}$ \\ ${ }^{1}$ European Science Communication Institute (ESCI), Lindenstraße 87, 26123 Oldenburg, Germany
}

\begin{abstract}
Social Media platforms are increasingly receiving attention from scholars, as they are presumed to be both useful tools for undertaking professional assignments and a medium for engaging with large audiences and communities, within and outside academia. Additionally, these novel practices online need proper assessment and evaluation procedures. This paper aims to address the possibilities and challenges for niche research and development $(R \& D)$ projects in communicating their research via social media. The authors applied a seven-step social media strategy to an ongoing energy efficiency case study and discuss an online tool for monitoring the respective impact on social media.
\end{abstract}

\section{Introduction. Scientists on Social Media}

Social Media (SM) platforms and, more broadly, digital online tools, have profoundly transformed the way in which science is performed. This revolution concerns all stages of the research process [1], from conceptualization to the dissemination of results, turning it into a more open, transparent and, to some extent, more social activity. Moed [2] argues that the research environment is undergoing a process of computerization, and that the incorporation of SM into the research lifecycle [3-5] is an additional aspect of the process. Some authors pinpoint increased research productivity and effectiveness as one of the prime benefits that SM brings to academia $[3,6]$.

SM is present and useful across the whole research process. It has strongly influenced science communication and the dissemination of research outcomes [7]. Conversations about science do not take place exclusively within the traditional channels (namely, scientific journals and conferences), but both online and offline communication activities can run in parallel, be combined or overlap to some extent.

In this context, one basic question needs to be answered: What is a SM platform? Sugimoto and colleagues [8] propose a categorization of SM platforms by keeping the focus on their use by scientists: social networking sites, social bookmarking and reference managers, video and media sharing platforms, social data sharing platforms, blogging, microblogging, wikis, and social recommending, rating and reviewing sites. Academic Social Networking Sites, such as ResearchGate or Academia.edu, come under the first category.

\footnotetext{
* Corresponding author: eb@esci.eu
} 
In terms of communication potential, the empirical study undertaken by Gruzd and Goertzen [9], revealed that the main benefits of using SM acknowledged by scientists themselves can be clustered into three main categories: "Information gathering"; "Collaboration"; and "Social \& information dissemination", the latter referring to the idea of self-promotion by making new contacts and vice versa. The categories "Information gathering" and "Collaboration" are mentioned much more frequently. This suggests that the uptake of SM occurs "more quietly and indirect[ly] at first" [10] integrating scientific working environments, and before moving to public discussions and outreach [10]. Nicholas and Rowland [3] argue that the scholarly use of these online resources constitutes a medium to construct public profiles and reputation and thus "impacting research in a number of key ways" [3] (see also [6, 11]).

In spite of its benefits, SM is far from being widely accepted among researchers. Figures show a moderate to very low adoption depending on the particular platform. Even when researchers have set up a profile on a given platform in order to be contacted, their use might not be very frequent $[12,13]$. Different reasons may explain such reluctance, but most of the literature points to a lack of time as the prime hurdle [12, 14]. Another important reason is the lack of knowledge about how to set up and properly use these tools [12], followed by a perceived uselessness $[6,7,14]$. On the whole, these low levels of adoption sharply contrast with the widespread awareness about the importance of SM among scholars [15].

\section{Developing a comprehensive SM strategy}

Collins and colleagues [12] present a lack of knowledge on how to use SM as one of the main obstacles that scientists must overcome in order to engage with these platforms. Therefore, some literature has tried to explain the technical and behavioural aspects around certain SM platforms to other scholars (see, for instance, [7], for an introduction to Twitter) or has attempted to write elaborate guidelines on facilitating the uptake and maximising the impact for researchers $[4,6,16]$.

Technological expertise on SM channels is undoubtedly important, but secondary to a communication strategy. Businesses have been using SM strategically for a long time in their overall marketing planning. Thackeray et al. [17] and Kietzmann et al. [18] argue that clear strategies help to maximise consumer impact and improve social marketing results, while Effing et al. [19] and Hallahan et al. [20] provide definitions and evaluations of strategic communication. Although the objective of communicating scientific research is not primarily focused on marketing principles, strategic SM planning will maximise impact.

\subsection{Principles of a good communication strategy}

The authors of this paper propose seven steps (Fig. 1) for maximising awareness and impact across various groups as part of a successful SM strategy for scientific or engineering projects such as those under the European Horizon 2020 (H2020) programme or nationally supported programmes:

1. Key target groups: Identify the potential audience and divide them into separate specific target groups.

2. Message: Communication should be tailored and fine-tuned to the specific target groups.

3. Media material: Messaging needs to draw on the most suitable media available, such as photographs, videos, info-graphics, plain text or a combination thereof. 
4. Communication channels: Depending on the media and target group, the most appropriate communication channels need to be selected for delivering the content.

5. Timing: All communication should reflect the actual state of the research activities. The following phases are suggested:

a) Phase 1 -Awareness Creation: Introducing target audiences to the subject of a research project with its scope and objectives.

b) Phase 2 -Information Sharing: Informing target audiences about the technological breakthroughs, potential business benefits or societal impact.

c) Phase 3 - Strategic Communication: Demonstrating how an innovation or "good" research results work, supporting exploitation and business models, and creating a public debate.

6. Partners network: Support research and technology partners in disseminating project results and benefit from their networks.

7. Monitoring tools. Use analytical tools, to ensure the project's communication and dissemination strategy delivers full impact.

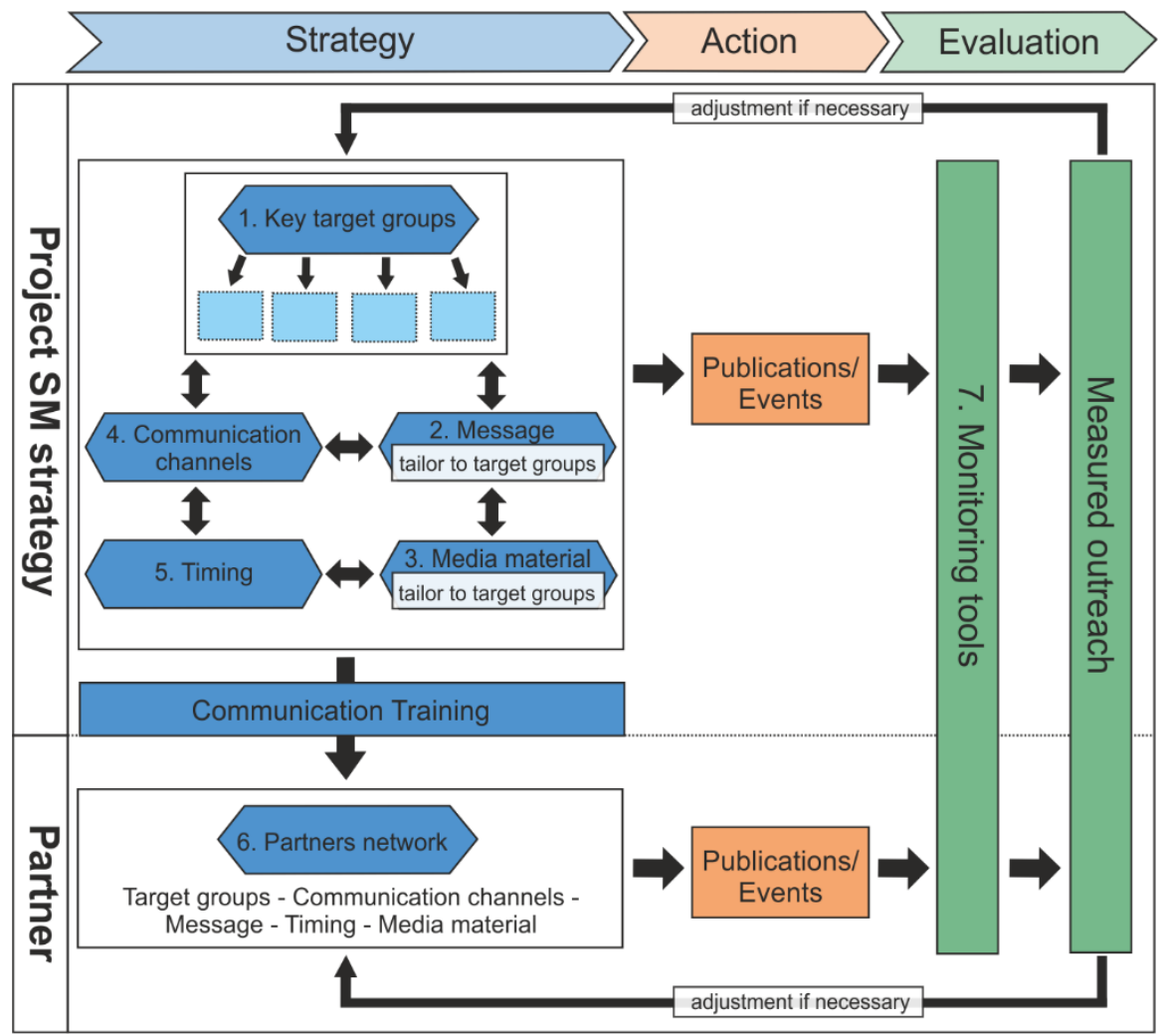

Fig. 1. Visualisation of the interaction and order of the seven steps of a SM strategy for a scientific or engineering endeavour.

\subsection{Communication strategy - a practical approach}

The authors of this paper are currently applying these seven steps to the EU-funded research project ETEKINA [21]. The project aims to recover 57-70\% of the waste heat stream in energy-intensive industries and brings together scientists and engineers from various 
industrial sectors. By adapting turnkey modular heat pipe heat exchanger (HPHE) technology to the aluminium, steel and ceramic sectors, it will make a business case for the proposed solutions in different sectors. The key target groups and key messages have been summarised in table 1 .

Table 1. Key target groups and key messages for the EU-funded research project ETEKINA.

\begin{tabular}{|c|c|}
\hline Key target groups & Key Messages \\
\hline $\begin{array}{l}\text { HPHE users, i.e. usually energy-intensive } \\
\text { industries. }\end{array}$ & $\begin{array}{c}\text { Return on investment (ROI) under three } \\
\text { years; reduced energy costs and improved } \\
\text { competitiveness }\end{array}$ \\
\hline HPHE producers & $\begin{array}{l}\text { Replicable solution; economic advantages; } \\
\text { access to potential clients }\end{array}$ \\
\hline Scientific community & $\begin{array}{l}\text { Access to design, data and results; } \\
\text { implications for future research }\end{array}$ \\
\hline $\begin{array}{c}\text { Policy makers and other stakeholders in } \\
\text { the fields of thermal recovery and energy- } \\
\text { intensive industries }\end{array}$ & $\begin{array}{l}\text { Comparison with other solutions; illustration } \\
\text { of benefits and impact }\end{array}$ \\
\hline Non-specialist audience; "general public" & $\begin{array}{l}\text { Reduction of } \mathrm{CO}_{2} \text { emissions; job security; } \\
\text { cheaper industry products }\end{array}$ \\
\hline
\end{tabular}

The authors raise awareness about ETEKINA via a full range of channels and they have produced infographics, brochures, roll ups, postcards, videos, photographs and GIFs, journalistic articles and interviews. The project has its own website and SM accounts (Twitter, YouTube, LinkedIn) where most of the material has been published. Material has also been published on multiplier platforms CORDIS [cordis.europa.eu], Alphagalileo [alphagalileo.org] as well as on partners' websites and within press releases.

This raises the question of whether such a seven-step SM campaign could also have a measurable impact for a research area with a small, well defined audience, where this audience may not be very active on SM. To answer this, the authors of this paper chose to assess the ETEKINA SM strategy with a state-of-the-art online monitoring tool.

\section{Monitoring SM activities}

There are many different commercial tools for analysing, monitoring and managing SM activity [22]. They have been designed primarily for marketing agencies or sales \& marketing departments of commercial companies. These companies generally target different groups than innovative energy efficiency projects do. It was therefore necessary to test various tools. Two were shortlisted: TalkWalker [talkwalker.com] and Digimind [digimind.com].

In two 4-week trials, they were both tested for relevance to the results of ETEKINA and other H2020 projects. First, the monitoring features were tested on the type of data actually collected and displayed, including geographical tagging, influencers networks, keywords and sentiments in individual posts. These tests were conducted for the various SM platforms, namely Twitter [twitter.com], LinkedIn [linkedin.com], Facebook [facebook.com], Instagram [instagram.com] and YouTube [youtube.com]. Eventually, Digimind was selected as the preferred monitoring tool.

\subsection{Digimind background}

Digimind self-defines as a SM analytics \& competitive intelligence tool which helps companies and brands to monitor their online impact by collecting information from a variety 
of sources, ranging from general outlets to social networking sites or blogs. It can also be run for benchmarking and business intelligence purposes, since it does not only track the interest around an organisation's own communication actions, but what competitors are doing as well.

This platform has two main functions. The Analytics module can automatically collect quantitative data of subscribers, interactions and publications (Fig. 2) from predefined SM platforms, like Twitter, YouTube and to a limited degree from LinkedIn. Digimind allows you to oversee the scope of your community of influence at a given time. Furthermore, once something is posted, it is possible to get immediate information about which and how many interactions the post is receiving. In this respect, it substitutes metrics already provided by single SM platforms, thus becoming useful when there are many of them mobilised at the same time. By grouping and scaling data from every single channel, Digimind brings quantitative insights on the overall SM performance.

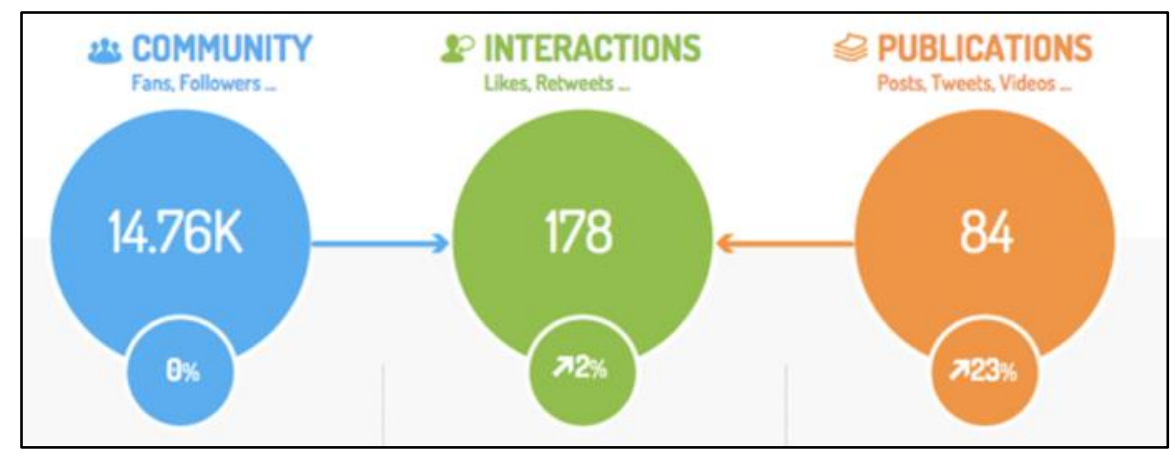

Fig. 2. Example of Analytics overview, showing the three main modules Community, Interactions and Publications; taken from digimind.com (13.03.2019; 10:00).

But the real potential of Digimind (as the rest of SM monitoring resources) resides in its Listening module, the very heart of the platform. This feature lets users unveil conversations about their brand, company, product or any other entity being monitored; regardless of how fragmented or dispersed they are online. Digimind crawls most of the web to retrieve those mentions that a given entity has received, indicating who has talked about it (influencers, demographics and outreach analysis); what has been said (content and sentiment analysis); when it has happened (time analysis); and where the mention has taken place (geographic and media breakdown).

Keyword combination is paramount in the Digimind's Listening module. Unleashing this enormous potential requires careful use of Boolean operators (i.e. OR, NOT and AND). Subsequently, this is the main drawback of Digimind as a monitoring tool: all its capabilities are grounded on a machine-based language. Depending on the keyword settings the results displayed can be incomplete or contain non-related mentions (noise).

\subsection{Evaluating ETEKINA's SM impact}

The monitoring of ETEKINA's SM impact started on 1 October 2018, twelve months into the project. In parallel, the monitoring of SM activities was also set up for other H2020 research projects, in which the European Science Communication Institute is involved. 


\subsubsection{Monitoring set-up}

Retrieving the mentions for a monitored entity is based on a set-up of keywords (see chapter 3.1), applying the Boolean operators to finetune the filters and minimise noise. The authors have found that in comparison to broader research projects (i.e. IRIS Smart Cities [23]), the monitoring of ETEKINA can be done without much noise. This is most likely due to its niche status in scientific research. The filter settings of the queries do not have to include many exceptional rules, as the keywords are clear and not often mistaken with another context.

Additionally, the name ETEKINA is not a homonym and this is a great help to monitoring. A project whose name has various meanings in other fields will be much harder to monitor as it produces more noise. The authors would therefore like to warn future project consortiums about this when choosing a project name.

\subsubsection{Influencer networks}

The analysis of the influencer networks of four research projects, namely ETEKINA, Laser4Surf [24], IRIS Smart Cities [23] and NextGen [25] shows how the impact on SM activities can differ significantly, depending on the nature of the project.

As Figure 3 shows, ETEKINA is influenced by mainly four partners - IK4-IKERLAN, University of Brunel, University of Modena and ESCI as well as individuals like Sam Jouhara, the project's technical director. However, participating small and medium-sized enterprises (SMEs) and industry partners show very little or no activity at all; none of the three industry partners even have a SM account, and SM plays virtually no role in their marketing activities.

When this data is compared with the influencer networks of other H2020 Projects, namely Laser4Surf (Fig. 4C), the pattern of SM interactions has a similar shape to ETEKINA's. This is probably because Laser4Surf is also a niche innovation initiative aimed at industry and is not directly relevant to the general public. In contrast, the IRIS Smart Cities and the NextGen influencer networks (Fig. 4A and B) are much larger. This can be partially explained by the fact that these projects have a lot more partners 43 (IRIS) and 30 (NextGen) compared to 8 (Laser4 Surf) and 10 in ETEKINA. Furthermore, the Key target groups and Message of the content are very different, as issues like innovative public transport, electric vehicles and new energy concepts for cities (IRIS) or water security (NextGen) already play an important role for citizens.

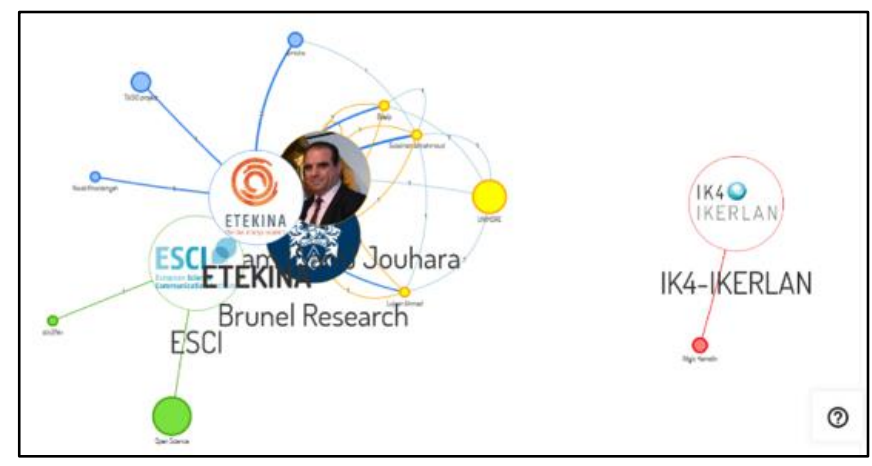

Fig. 3. Overview of the influencer network for the ETEKINA project; timeframe: 1 Nov 2018 to 10 March 2019; source: Digimind Listening module. 


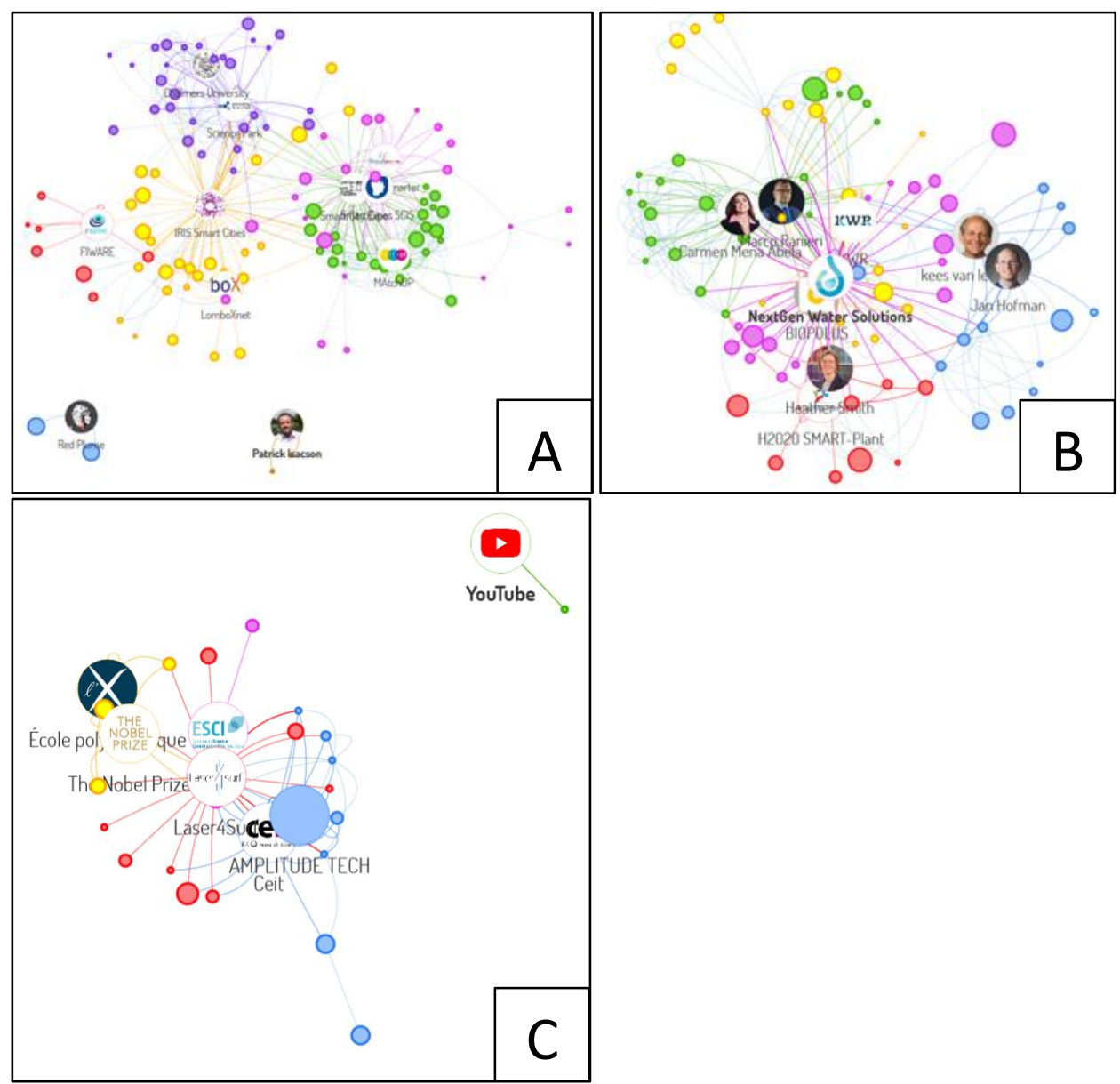

Fig. 4. Influencer network of IRIS Smart Cities[A], NextGen [B] and Laser4Surf [C], timeframe 1 Nov 2018 to 10 March 2019; source: Digimind Listening module.

Those partners who stand to gain most from ETEKINA's results are expected to become more active on SM once the results start to emerge. The (influencer) network is expected to expand organically, although the authors suggest strategic support from the project's communication officer. ETEKINA's communication officer has recently joined special interest groups on LinkedIn, such as the Heat Exchanger and Heat Transfer Group (with 5190 members) and the Waste Heat Recovery group (with 947 members). Potential influencers outside the reach of project partners can thus be approached.

Clearly, the project network can also be expanded by offline activities such as attending conferences, fairs and other business events. These currently help industrial partners and small businesses involved in $\mathrm{H} 2020$ project in their growth.

Nevertheless, the authors encourage those partners who are inactive on SM to go on SM courses to support their exploitation activities. 


\section{Conclusion}

SM platforms transform and shape today's research environment and will continue to do so in the future. Scholars strive to create meaningful impact and visibility for their research. To achieve this, they need training in SM to better understand its pitfalls and opportunities. Effective science communication on SM starts with a good communication strategy. The authors propose a seven-step framework for a successful SM campaign, based on Key target groups, Message, Media material, Communication channel, Timing, Partners network and Monitoring tools.

To keep track on impacts achieved by communication activities, the authors argued that commercial monitoring tools could be used even for special interest R\&D projects. During the monitoring process, the authors discovered, that while niche projects benefit from being well defined compared to broader projects, it is harder to reach a wider audience and maximise impact via SM.

Finally, the authors propose that outreach via SM could complement, with never completely replacing more direct outreach and engagement activities [13].

This work was co-funded by the innovation action ETEKINA (HEAT PIPE TECHNOLOGY FOR THERMAL ENERGY RECOVERY IN INDUSTRIAL APPLICATIONS) of the European Union's Horizon 2020 research and innovation programme under the grant agreement $\mathrm{N}^{\circ} 768772$.

\section{References}

1. European Commission, Open Innovation, Open Science, Open to the World - a vision for Europe (Publications Office of the European Union, Luxembourg, 2016)

2. F. M. Moed, [in:] C. R. Sugimoto (ed.), Theories of Informetrics and Scholarly Communication: A Festschrift in honor of Blaise Cronin (De Gruyter Saur, Berlin-Boston, 2017)

3. D. Nicholas, I. Rowland, Inf. Serv. \& Use 31, 61-83 (2011)

4. I. M. Côté, E. S. Darling, Facets 3, 682-694 (2018)

5. E. S. Darling, D. Shiffman, I. M. Côté, J. A. Drew, Peer J. PrePrints, 1 (2013)

6. H. M. Bik, M. C. Goldstein, PLoS Bio. 11, 4 (2013)

7. K. Holmberg, M. Thelwall, Scient. 101, 2, 1027-1042 (2014)

8. C. R. Sugimoto, S. Work, V. Larivière, S. Haustein, JASIST 68, 9, 2037-2062 (2017)

9. A. Gruzd, M. Goertzen, $46^{\text {th }}$ Hawaii International Conference on System Sciences, 3332-3341 (2013)

10. T. McCormick, Elsevier Connect (2014). Retrieved from:

https://www.elsevier.com/connect/social-media-for-scientists-including-the-shyovercommitted-and-unconvinced

11. H. M. Bik, A. D. M. Dove, M. C. Goldstein, R. R. Helm, R. MacPherson, K. Martini, A. Warneke, C. McClain, PLoS Comp. Bio. 11, 4 (2015)

12. K. Collins, D. Shiffman, J. Rock, PLoS One 11, 10 (2016)

13. R. van Noorden, Retrieved from: https://www.nature.com/news/online-collaborationscientists-and-the-social-network-1.15711 (Nature, 2014)

14. L. McHeyzer-Williams, M. McHeyzer-Williams, Tr. in Immun. 37, 4, 260-265 (2016)

15. C. MacClain, L. Neely, F100 Res. 3 (2014) 
16. N. A. Lewis, J. J. van Bavel, L. H. Somerville, J. Gruber, Retrieved from: https://www.sciencemag.org/careers/2018/11/social-media-survival-guide-scientists (Science, 2018)

17. R. Thackeray, B. L. Neiger, H. Keller, Health promotion practice 13, 2, 165-168 (2012)

18. J. H. Kietzmann, K. Hermkens, I. P. McCarthy, B. S. Silvestre, Business horizons 54, 3, 241-251 (2011).

19. R. Effing, T. A. Spil, International Journal of Information Management 36, 1, $1-8(2016)$

20. K. Hallahan, D. Holtzhausen, B. Van Ruler, D. Verčič, K. Sriramesh, International Journal of Strategic Communication 1, 1, 3-35 (2007)

21. ETEKINA - heat pipe technology for thermal energy recovery in industrial applications; Grant agreement ID: 768772; Project Coordinator: IKERLAN S COOP; (https://cordis.europa.eu/project/rcn/212362/factsheet/en; 14.03.2019; 16:30)

22. S. Chen, L. Lin, X. Yuan, Computer Graphics Forum 36, 3, 563-587 (2017)

23. IRIS - Integrated and Replicable Solutions for Co-Creation in Sustainable Cities; Grant agreement ID: 774199; Project coordinator: GEMEENTE UTRECHT (https://cordis.europa.eu/project/rcn/212411/factsheet/en; 14.03.2019, 16:30)

24. Laser4Surf - laser for mass production of functionalised metallic surfaces; Grant agreement ID: 768636; Project coordinator: ASOCIACION CENTRO TECNOLOGICO CEIT-IK4 (https://cordis.europa.eu/project/rcn/211229/factsheet/en; 14.03.2019, 16:30)

25. NextGen - Towards a next generation of water systems and services for the circular economy; Grant agreement ID: 776541; Project Coordinator: KWR WATER B.V. (https://cordis.europa.eu/project/rcn/216646/factsheet/en; 14.03.2019; 16:30) 